\title{
Pulsed Laser Polymerization at Low Conversions: Broadening and Chain Transfer Effects
}

\author{
BEN O'SHAUGHNESSY* and DIMITRIOS VAVYLONIS \\ Department of Chemical Engineering \\ Columbia University \\ 500 West 120th Street \\ New York, NY 10027, USA
}

\author{
This is a preprint of an article accepted for publication in \\ Macromolecular Theory and Simulations \\ (c) 2003 Wiley-VCH Verlag GmbH \\ * To whom correspondence should be addressed.
}




\section{SUMMARY}

Pulsed laser polymerization (PLP) is widely employed to measure propagation rate coefficients $k_{p}$ in free radical polymerization. Various properties of PLP have been established in previous works, mainly using numerical methods. The objective of this paper is to obtain analytical results. We obtain the most general analytical solution for the dead chain molecular weight distribution (MWD) under low conversion conditions which has been hitherto obtained. Simultaneous disproportionation and combination termination processes are treated. The hallmarks of PLP are the dead MWD discontinuities located at integer multiples of $n_{0}=k_{p} t_{0} C_{\mathrm{M}}$, where $t_{0}$ is the laser period and $C_{\mathrm{M}}$ is the monomer concentration. We show that chain transfer reduces their amplitude by factors $e^{-c_{t r} L n_{0}}$, consistent with numerical results obtained by other workers. Here $c_{t r}$ is the chain transfer coefficient and $L n_{0}(L=$ integer) are the discontinuity locations. Additionally, transfer generates a small amplitude continuous contribution to the MWD. These results generalize earlier analytical results which were obtained for the case of disproportionation only. We also considered 2 classes of broadening: (i) Poisson broadening of growing living chains and (ii) intrinsic broadening by the MWD measuring equipment (typically gel permeation chromatography, GPC). Broadening smoothes the MWD discontinuities. Under typical PLP experimental conditions, the associated inflection points are very close to the discontinuities of the unbroadened MWD. Previous numerical works have indicated that the optimal procedure is to use the inflection point to infer $k_{p}$. We prove that this is a correct procedure provided the GPC resolution $\sigma$ is better than $n_{0}^{1 / 2}$. Otherwise this underestimates $L n_{0}$ by an amount of order $\sigma^{2} / n_{0}$.

Keywords: molecular weight distribution/propagation rate coefficients/pulsed laser polymerization/radical polymerization/theory 


\section{Introduction}

A fundamental material parameter in free radical polymerization (FRP) is the propagation rate constant 1123 $k_{p}$ governing the sequential addition of monomers to the active free-radical ends of "living" chains (see fig. (1). In this paper we consider theoretically "pulsed laser polymerization" (PLP), 45 possibly the most accurate method to to measure $k_{p}$. In PLP new radicals (living chains of one monomer, $N=1$ ) are generated by a sequence of laser pulses which photocleave photoinitiators in the reaction mix. The laser is flashed periodically (the duration of each flash is extremely short (see fig. 5(a))) with flash period $t_{0} \sim 0.1 \mathrm{sec}$ optimally chosen to be somewhat shorter than the average living chain lifetime. Thus every $t_{0}$ the living chain population is updated by the injection of new primary radicals. After many cycles a periodic state is established where the time averaged rate of radical production balances the time averaged rate of living chain termination: pairs of living chains terminate to generate either a single ("combination") or a pair ("disproportionation") of "dead" chains, the final polymer product (see fig. 2).

Now unlike the dead molecular weight distribution (MWD) of standard steady state FRP which has no singular features, in the case of PLP the dead MWD, $\phi_{\text {dead }}(N)$, is predicted theoretically to possess finite discontinuities $\frac{36}{6}$ at chain lengths $N=L n_{0}$, where $L=0,1,2, \ldots$ and

$$
n_{0} \equiv v_{p} t_{0}, \quad v_{p} \equiv k_{p} C_{\mathrm{M}}
$$

Here $n_{0}$ is the number of monomers added to a growing living chain in time $t_{0}$ and the "propagation velocity" $v_{p}$, namely the rate of monomer addition to a growing chain, is proportional to the monomer concentration $C_{\mathrm{M}}$. The origin of the dead MWD discontinuities is the coherent sudden increase in termination rates due to the sudden increase in radical population following each pulse. In principle the discontinuities can be seen experimentally after measuring the resulting MWD by gel permeation chromatography (GPC). Thus $n_{0}$ and hence $v_{p}$ (or equivalently $k_{p}$ ) is inferred. This method has been employed to deduce $k_{p}$ for a variety of polymerizing systems. 789101112

In reality there exist a number of practical difficulties. (1) Broadening effects. Consider a living chain created at $t=0$. After time $t_{0}$ its length is not exactly $v_{p} t_{0}$; since polymerization is a random process, Poisson fluctuations arise around this length. This then leads to a softening of the theoretically infinitely sharp dead MWD discontinuities. Another, but quite different effect, is the direct broadening of the dead MWD due to intrinsic uncertainties in the measuring method. GPC equipment if supplied with a discontinuous dead MWD will output a reading indicative of a somewhat broadened discontinuity due to fluctuations in the transit time of a chain of a given length through the gel. (2) Chain transfer ${ }^{1}$ of radicals from living chain ends to surrounding unpolymerized monomer (fig. 3), quantified by the parameter $\lambda$. This is simply related to the chain transfer coefficient $c_{t r}$ :

$$
c_{t r} \equiv \frac{k_{t r} C_{\mathrm{X}}}{k_{p} C_{\mathrm{M}}}=\frac{\lambda}{v_{p}},
$$

where $k_{t r}$ is the 2 nd order rate constant governing chain transfer to species $\mathrm{X}$ whose concentration is $C_{\mathrm{X}}$ ( $\mathrm{X}$ is usually the monomer species). One expects chain transfer, important for species such as vinyl acetate and polystyrene, 1113 to dephase the living MWD which would otherwise be a sequence of in-phase pulses. This will then affect the discontinuities in the dead MWD.

Typical experimental MWDs produced by PLP are shown in fig. 廿 where an obviously broadened first discontinuity is evident near molecular weight 40,000. In order to deduce the correct propagation rate constant, it is essential to have an algorithm to infer $n_{0}$ from such a broadened discontinuity. Taking $n_{0}$ as the molecular weight corresponding to the lower point of inflection, near the middle of the broadened discontinuity, has been proposed as the optimal approach.6 14 Other authors have advocated that under particular conditions this criterion should be replaced with the maximum point. 1516

In order to design and interpret PLP experiments many researchers have employed numerical simulations of PLP chemical kinetics. By varying the parameters of the simulations the resulting dead MWD is modeled under different experimental conditions. In such numerical studies Poisson-broadening is automatically taken into account and numerous side-reactions such as chain transfer can be incorporated. Guidelines can then be derived for extracting $k_{p}$ from the broadened MWD peak. Such numerical approaches have so far been employed both in the absence 111718 and the presence of chain transfer.131916 The effects of experimental broadening have been addressed numerically in refs.17 16

Analytical solutions of PLP dynamics are also very desirable. Where available they provide a systematic and quantitative framework which exactly articulates the conclusions of the underlying physical model, 
beyond the scope of numerical analyses. Analytical expressions can also greatly facilitate experimental design by obviating the need for numerical work. The first analytical solution of PLP dynamics appeared in refs. 620 and included the effects of Poisson broadening for termination by both combination and disproportionation. A new derivation and a review of these theoretical results is given in ref. 21] A closed form for the dead MWD for arbitrary sequences of laser pulses was derived in ref. 22 Analytical work including chain transfer exists for the case of termination by disproportionation only. 2324

In this paper we develop a completely general analytical theory for PLP, including the effects of broadening and chain transfer analytically. We thus quantify the effect of these two mechanisms on the theoretically infinitely sharp dead MWD which enables us to discuss the validity of empirical rules on deriving $k_{p}$ from the broadened MWD peak. We consider a general (and typical) situation where both combination and disproportionation events occur. This extends previous analytical studies. We derive the most general analytical expressions which have been obtained hitherto for PLP, to the best of our knowledge. Closed expressions are derived in terms of the following independent parameters: $v_{p}, k_{c}, k_{d}, \lambda, t_{0}$, and the concentration of radicals produced per laser pulse. Here $k_{c}$ and $k_{d}$ are the combination and disproportionation rate constants, respectively (see fig. 2). An important quantity in what follows is the net termination rate constant,

$$
k \equiv k_{c}+k_{d} .
$$

Our analysis describes low conversion FRP which allows us to make the approximation that the net livingliving bimolecular termination rate constant, $k$, is independent of chain length (see fig. 2). In reality $k(M, N)$ depends on the degrees of polymerization $M, N$ of the reacting chains. However in dilute solutions (under good solvent conditions) theory ${ }^{252627}$ predicts a very weak dependence on chain lengths: $k(N, N) \sim N^{-\alpha}$ with $\alpha \approx 0.16$. A very weak dependence of $k$ on chain lengths has also been well established experimentally in phosphorescence quenching studies. $\frac{28}{28}$ At low conversions, living chain terminations occur in a pure monomer solvent (usually a good one) and thus the approximation of constant $k$ is expected to yield rather accurate conclusions. Making this approximation, all quantities can be calculated exactly. The effect of the dependence of termination rates on chain length has been addressed numerically in refs. 29303132333418 which employed various empirical rules for the form of $k(M, N)$.

The living MWD, $\phi(N, t)$, like all quantities settles down to a periodic form with period $t_{0}$. Bimolecular reactions as in figs. 20 generate dead chains of length $N$ at a rate

$$
\dot{\phi}_{\text {dead }}(N, t)=\frac{1}{2} k_{c} \int_{0}^{N} d M \phi(M, t) \phi(N-M, t)+k_{d} \phi(N, t) \Psi(t)+\lambda \phi(N, t),
$$

where $\Psi$ is the total concentration of living chains. The resulting dead MWD will include Poisson broadening effects provided one uses the correct Poisson broadened living MWD in eq. (4). In the following we will employ the notation $\phi_{\text {dead }}$ to denote the dead MWD without Poisson broadening, including however chain transfer effects (i. e. $\phi_{\text {dead }}$ results from using in eq. (4) the form for $\phi(N, t)$ which correctly includes chain transfer but which neglects Poisson broadening). The full Poisson broadened MWD is denoted $\phi_{\text {dead }}^{\text {broad }}$ while the reading output by the GPC measuring equipment we name $\phi_{\text {dead }}^{\text {read }}$.

The dead MWD is calculated in successive steps as follows. In section 2 we review "ideal" PLP, i.e. we ignore chain transfer (setting $\lambda=0$ ) and broadening effects. The time averaged dead MWD, $\phi_{\text {dead }}^{\text {ideal } \text {, turns }}$ out to have finite discontinuities at $N=j n_{0}$ ( $j$ is a positive integer). In section 3 we generalize the results of section 2 to include chain transfer and express the resulting dead MWD, $\phi_{\text {dead }}$, in terms of $\phi_{\text {dead }}^{\text {ideal }}$. We find that chain transfer diminishes the amplitude of the dead MWD discontinuities, but does not broaden them. Broadening effects due to both Poisson broadening and due to limited experimental resolution are included in section 4 . We show that the inflection point rule is correct for inferring $n_{0}$ only when the width of the Poisson broadened discontinuity is larger than the experimental resolution. We conclude with a summary of our results in section 5 .

Throughout, $[N]$ will denote the nearest integer to $N / n_{0}$ which is less than $N / n_{0}$, whilst $r N \equiv \operatorname{rem}\left[N, n_{0}\right]$ denotes the remainder of $N$ modulo $n_{0}$. Similar notation will apply to time $t$, with $n_{0}$ replaced by $t_{0}$. Thus

$$
N \equiv[N] n_{0}+r N, \quad r N \equiv \operatorname{rem}\left[N, n_{0}\right] ; \quad t \equiv[t] t_{0}+r t, \quad r t \equiv \operatorname{rem}\left[t, t_{0}\right] .
$$




\section{Ideal PLP}

Let us first briefly review the "ideal" PLP problem ignoring the effects of broadening and chain transfer, as analyzed by Olaj et al. 621 Termination may occur either by combination or disproportionation reactions. First we calculate the living MWD which depends on the total termination rate constant, $k$, only, and not on the mode of termination. We then derive separate expressions for the dead chains terminated by combination and disproportionation, respectively.

\subsection{Total Concentration of Living Chains $\Psi(t)$ and Reaction Field $H(t)$}

The number density MWD of living chains, $\phi(N, t)$, obeys

$$
\dot{\phi}=-v_{p} \phi^{\prime}-H(t) \phi+\delta(N) R_{i}(t)
$$

with $\phi(N<0, t)=0$. Here $\dot{\phi} \equiv \partial \phi / \partial t$ and $\phi^{\prime} \equiv \partial \phi / \partial N$. Note that the $\delta(N) R_{i}(t)$ term which injects primary radicals (chains of length $N=0$ in our continuous framework) into the system at rate $R_{i}(t)$ per unit volume, is equivalent to the boundary condition $v_{p} \phi(0, t)=R_{i}(t)$. In PLP dynamics the time-dependent primary radical production rate, $R_{i}(t)$, is a train of pulses,

$$
R_{i}(t)=\bar{R}_{i} t_{0} \sum_{j=-\infty}^{\infty} \delta\left(t+j t_{0}\right)
$$

as shown in fig. 5(a). $\bar{R}_{i}$ is the time averaged radical production rate. The $-v_{p} \phi^{\prime}$ term in eq. (6) describes propagation whilst $H(t) \phi(N, t)$ is the total termination rate (due to both combination and disproportionation) for a chain of length $N$ due to the "reaction field" $H(t)$, namely the total termination rate per unit time for a given chain due to all other chains in the system:

$$
H(t) \equiv k \Psi(t), \quad \Psi(t) \equiv \int_{0}^{\infty} d N \phi(N, t)
$$

Here $\Psi(t)$ is the total number density of living chains whose dynamics follow after integrating eq. (6) over all positive $N$ values:

$$
\dot{\Psi}=-H(t) \Psi+R_{i}(t)=-k \Psi^{2}+R_{i}(t) .
$$

For $t$ different from integer multiples of $t_{0}$ one has $R_{i}(t)=0$ and thus $\Psi$ obeys simple second order reaction kinetics, $\dot{\Psi}=-k \Psi^{2}$, with solution

$$
\Psi(t)=\frac{\Psi_{0}}{1+t k \Psi_{0}} \quad\left(0<t<t_{0}\right),
$$

where $\Psi_{0} \equiv \Psi\left(0^{+}\right)$is the value of $\Psi$ just after a new laser pulse at $t=0$. We define $\beta$ as the surviving fraction just before the next pulse at $t_{0}$,

$$
\beta \equiv \Psi\left(t_{0}^{-}\right) / \Psi_{0}
$$

The values of $\Psi_{0}$ and $\beta$ when the system settles down to a periodic stationary state are determined in terms of $\bar{R}_{i}, k$, and $t_{0}$, by equating the number of terminated chains in one cycle with the number of new living chains introduced by a pulse,

$$
(1-\beta) \Psi_{0}=\bar{R}_{i} t_{0}
$$

This relation together with eqs. (10) and (11) yields

$$
\Psi_{0}=\frac{1}{2} \bar{R}_{i} t_{0}\left[1+\left\{1+4 /\left(\bar{R}_{i} k t_{0}^{2}\right)\right\}^{1 / 2}\right], \quad \beta=\frac{1}{1+k \Psi_{0} t_{0}} .
$$

The steady state $\Psi(t)$, sketched in fig. [5(c), is of course periodic with period $t_{0}$, and has discontinuous jumps $\Delta \Psi=(1-\beta) \Psi_{0}$ at integer multiples of $t_{0}$. 


\subsection{Living MWD}

The solution to eq. (6) is derived in appendix A for a general $R_{i}(t)$ in terms of $H(t)$ whose important properties are derived in appendix B. Using the present periodic form for $R_{i}(t)$, eq. (7), using eqs. (12) and (B2) and recalling the periodicity of $\Psi$ and $H$, we have

$$
\begin{aligned}
\phi(N, t) & =(1-\beta) \Psi_{0} \Theta(N) \sum_{j=-\infty}^{\infty} e^{-\int_{0}^{N / v_{p}} H\left(t^{\prime}\right) d t^{\prime}} \delta\left(N-v_{p} t-j n_{0}\right) \\
& =\sum_{j=-\infty}^{\infty} \phi_{e n v}(N) \delta\left(N-v_{p} t-j n_{0}\right)
\end{aligned}
$$

where $\Theta$ is the step function $(\Theta(x>0)=1, \Theta(x<0)=0)$ and the function $\phi_{\text {env }}$, sketched in fig. 国 (b), is the living MWD envelope

$$
\phi_{e n v}(N) \equiv \Theta(N) \beta^{[N]}(1-\beta) \Psi\left(N / v_{p}\right)=\Theta(N) \beta^{[N]}(1-\beta) \frac{\Psi_{0}}{1+k \Psi_{0} r N / v_{p}} .
$$

The living MWD, sketched in fig. [5(b), is a train of pulses in phase with $R_{i}(t)$. A new $\delta$-pulse is born at $N=0$ at time $t=0$, say, and then moves in the $+N$ direction with velocity $v_{p}$. When in the interval $(j-1) n_{0}<N \leq j n_{0}$ (the " $j$ th sector") this pulse suffers the same field $H(t)$ as in all other sectors. The amplitude of the $\delta$-pulse in sector $j+1$ is thus reduced by a factor $\beta$ with respect to sector $j$ and follows the envelope $\phi_{e n v}(N)$.

\subsection{Dead MWD}

The time-averaged rate of dead chain generation, $\bar{\phi}_{\text {dead }}^{\text {ideal }}(N)$, is the sum of the contributions due to termination by combination and disproportionation, $\bar{\phi}_{\text {dead }}^{\text {deal }}(N)=\bar{\phi}_{\text {dead }}^{\text {comb }}(N)+\bar{\phi}_{\text {dead }}^{\text {disp }}(N)$. Each of these two contributions can be found by inserting eq. (14) into the first and second terms on the rhs of eq. (44), respectively, and then time averaging over one period. In the following we will use however a simpler method.

Consider first chains terminated by combination. We notice that, due to periodicity, the total concentration of dead chains of length $N$ produced by combination per period, $\bar{\phi}_{\text {dead }}^{\text {comb }}(N) t_{0}$, equals one half the concentration of dead chains produced by combination by one single $\delta$ pulse throughout its entire lifetime. Thus choosing the $j=0$ pulse in eq. (14), bimolecular kinetics imply

$$
\overline{\dot{\phi}}_{\text {dead }}^{\text {comb }}(N)=\frac{k_{c}}{2 t_{0}} \int_{-\infty}^{\infty} d t \int_{-\infty}^{\infty} d M \phi_{\text {env }}(M) \delta\left(M-v_{p} t\right) \phi(N-M, t) .
$$

This is proved rigorously in Appendix C. Since the integrand of eq. (16) is zero for negative $t$ and $M$ values, we have extended the lower limits of the integrals from 0 to $-\infty$ for future convenience.

Substituting the expression for $\phi$ of eq. (14) into the above expression, it is shown in Appendix D this leads to

$$
\bar{\phi}_{\text {dead }}^{\text {comb }}(N)=C \frac{k_{c}}{k}\left\{(L+1) \beta^{L} f^{2}\left(\frac{r N}{2}\right)+L \beta^{L-1} f^{2}\left(\frac{r N+n_{0}}{2}\right)\right\},
$$

where

$$
N \equiv L n_{0}+r N, \quad f(n) \equiv \frac{\beta}{\beta+(1-\beta) n / n_{0}}, \quad C \equiv \frac{k \Psi_{0}^{2}(1-\beta)^{2}}{4 v_{p} t_{0}} .
$$

Similarly, the time-averaged concentration of dead chains generated by disproportionation can be calculated by noting that $\bar{\phi}_{\text {dead }}^{\text {disp }}(N) t_{0}$ is equal to the concentration of dead chains of length $N$ produced by disproportionation by one single $\delta$-pulse throughout its lifetime. Choosing $j=0$ for this pulse in eq. (14) one has

$$
\overline{\dot{\phi}}_{\text {dead }}^{\text {disp }}(N)=\frac{k_{d}}{t_{0}} \int_{-\infty}^{\infty} d t \phi_{\text {env }}(N) \delta\left(N-v_{p} t\right) \Psi(t)
$$


Integrating the $\delta$ function in eq. (19) and using eq. (15) to express $\phi_{\text {env }}$ in terms of $\Psi$ yields

$$
\bar{\phi}_{\text {dead }}^{\text {disp }}(N)=4 C \frac{k_{d}}{k}\left\{\frac{\beta^{L}}{1-\beta} f^{2}(r N)\right\}, \quad N \equiv L n_{0}+r N
$$

The dead MWD, $\phi_{\text {dead }}^{\text {ideal }}=t \bar{\phi}_{\text {dead }}^{\text {ideal }}=t\left(\bar{\phi}_{\text {dead }}^{\text {comb }}+\bar{\phi}_{\text {dead }}^{\text {disp }}\right)$, is shown in fig. 5 (d). Both combination and disproportionation contributions have discontinuities at the end of each sector $L$ (where $L=0$ labels the first sector, $0 \leq N \leq n_{0}$ ). The discontinuity, $\Delta \phi_{\text {dead }}^{\text {ideal }}$, at the boundary of sectors $L-1$ and $L$ is

$$
\frac{\Delta \phi_{\text {dead }}^{\text {ideal }}\left(L n_{0}\right)}{\phi_{\text {dead }}^{\text {ideal }}(0)} \equiv \frac{\phi_{\text {dead }}^{\text {ideal }}\left(L n_{0}^{+}\right)-\phi_{\text {dead }}^{\text {ideal }}\left(L n_{0}^{-}\right)}{\phi_{\text {dead }}^{\text {ideal }}(0)}=\beta^{L} \frac{2+4 \rho}{1+4 \rho /(1-\beta)}, \quad \rho \equiv k_{d} / k_{c}
$$

\section{Chain Transfer}

In this section we will study how chain transfer modifies the results of section 2. Each transfer event results in one dead chain of length $N$ and one living chain of length $N=0$ as depicted in fig. 3

\subsection{Living MWD}

The living dynamics now include additional loss through transfer from chains of length $N$. These radicals are reinjected as a source term at $N=0$ :

$$
\dot{\phi}=-v_{p} \phi^{\prime}-[H(t)+\lambda] \phi+\left[R_{i}(t)+\lambda \Psi(t)\right] \delta(N)
$$

The total reinjection rate is $\lambda \Psi$ where $\lambda$ is defined in terms of the chain transfer coefficient in eq. (2).

Integrating eq. (22) over all positive $N$ values ( $\phi$ is zero for negative $N$ ) one immediately sees that the total number density of living chains $\Psi(t)$ obeys the same dynamics as without chain transfer, eq. (9). This must be true, of course, since transfer events conserve the total number of living chains and $k$ is independent of chain length in the present approximation. The crucial point is that $\Psi(t)$ and $H(t)=-k \Psi(t)$ appearing in eq. (22) are the same periodic functions of time as without chain transfer, eq. (10).

Since $H(t)$ is a known function of time, the dynamics of eq. (22) are effectively linear and we can use the superposition principle to write $\phi=\phi_{c o h}+\phi_{i n c}$ where

$$
\begin{aligned}
& \dot{\phi}_{c o h}=-v_{p} \phi_{c o h}^{\prime}-[H(t)+\lambda] \phi_{c o h}+R_{i}(t) \delta(N) \\
& \dot{\phi}_{i n c}=-v_{p} \phi_{i n c}^{\prime}-[H(t)+\lambda] \phi_{i n c}+\lambda \Psi(t) \delta(N) .
\end{aligned}
$$

Here $\phi_{c o h}$ is the coherent part, which as we will immediately see is a sum of pulses in phase with radical production, whilst $\phi_{i n c}$ is the incoherent part which is out of phase due to random transfer events.

Replacing $H \rightarrow H+\lambda$ in the general solution, eq. (A3), to the living dynamics without chain transfer, one sees $\phi_{c o h}$ is $e^{-\lambda N / v_{p}}$ times its no-transfer form of eq. (14). Similarly replacing $H \rightarrow H+\lambda$ and $R_{i} \rightarrow \lambda \Psi$, one obtains $\phi_{\text {inc }}$ after using eq. (B2). One finds:

$$
\begin{array}{ll}
\phi_{c o h}(N, t)=e^{-\lambda N / v_{p}} \sum_{j=-\infty}^{\infty} \phi_{e n v}(N) \delta\left(N-v_{p} t-j n_{0}\right), & \Psi_{c o h}(t)=\Psi(t) \frac{(1-\beta) e^{-\lambda t}}{1-\beta e^{-\lambda t_{0}}} \\
\phi_{i n c}(N, t)=\frac{\lambda e^{-\lambda N / v_{p}}}{v_{p}} \Psi(t) \beta^{[t]-\left[t-N / v_{p}\right]} \Theta(N), & \Psi_{i n c}(t)=\Psi(t)-\Psi_{c o h}(t),
\end{array}
$$

where $\Psi_{c o h}, \Psi_{i n c}$ are the corresponding total concentrations of chains in each part of the MWD. $\phi_{i n c}$ is a series of propagating pulses each of width $n_{0}$, the amplitude of which decreases with molecular weight and time. Notice however that unlike $\phi_{c o h}, \phi_{i n c}$ extends over all $N$ values at a given time. $\phi_{i n c}$ has discontinuities at $N=v_{p} t+j n_{0}$. At each discontinuity, $\phi_{i n c}$ decreases suddenly by a factor $\beta$. 


\subsection{Dead MWD}

Dead chains are generated by both living chain termination (first two terms on the rhs of eq. (4)) and chain transfer events (last term of eq. (4)). We consider first the contribution due to termination by combination. Using the results of Appendix $\mathrm{C}$, the time averaged rate of production of dead chains by combination is given by

$$
\bar{\phi}_{\text {dead }}^{\text {comb }}(N)=\frac{k_{c}}{2 t_{0}} \int_{-\infty}^{\infty} d t \int_{-\infty}^{\infty} d M e^{-\lambda M / v_{p}} \phi_{\text {env }}(M) \delta\left(M-v_{p} t\right)\left\{\phi_{\text {coh }}(N-M, t)+2 \phi_{\text {inc }}(N-M, t)\right\}
$$

This is the contribution to $\overline{\dot{\phi}}_{\text {dead }}$ due to termination by combination by one single $\delta$ pulse belonging to the coherent living MWD, throughout its lifetime. Combination occurs either with other coherent chains or by "cross-coupling" with chains belonging to $\phi_{i n c}$. In eq. (25) we have assumed that chain transfer is a weak effect, i. e. that $\lambda$ is small in a sense to be quantified below, and we keep the leading correction only. Thus we discarded the $\mathcal{O}\left(\lambda^{2}\right) \phi_{\text {inc }} \phi_{\text {inc }}$ termination term.

Now the coherent-coherent coupling term in eq. (25) is equal to that of eq. (16), multiplied by $e^{-\lambda N / v_{p}}$. The only difficulty is the coherent-incoherent cross-term $\bar{\phi}_{\text {dead,cross }}^{\text {comb }}$. In Appendix E it is shown that

$$
\begin{aligned}
\bar{\phi}_{\text {dead }, \text { cross }}^{\text {comb }}(N)=\frac{\lambda e^{-\lambda N / v_{p}}}{v_{p} t_{0}} \frac{k_{c}}{k}(1-\beta) \quad & \left\{\beta^{L}[(L+1) \beta-L] \Psi_{0}+\beta^{L}(L+1)(1-\beta) \Psi\left(\frac{r N}{2 v_{p}}\right)+\right. \\
& \left.L \beta^{L-1}(1-\beta) \Psi\left(\frac{r N+n_{0}}{2 v_{p}}\right)-\beta^{L} \Psi\left(\frac{r N}{v_{p}}\right)\right\} .
\end{aligned}
$$

Let us calculate now the disproportionation and chain transfer contributions, $\bar{\phi}_{\text {dead }}^{\text {disp }}$ and $\bar{\phi}_{\text {dead }}^{\text {trans }}$. Time averaging the last two terms of eq. (4) one has (bar denotes time average)

$$
\overline{\dot{\phi}}_{\text {dead }}^{\text {disp }}(N)+\overline{\dot{\phi}}_{\text {dead }}^{\text {trans }}(N)=k_{d} \overline{\phi(N) \Psi}+\lambda \bar{\phi}(N)
$$

Now from eq. (22) one has $k \overline{\phi(N) \Psi}+\lambda \bar{\phi}(N)=-v_{p} \partial \bar{\phi} / \partial N$, using $\bar{\phi}=0$ in stationary state. Thus using eq. (27),

$$
\overline{\dot{\phi}}_{\text {dead }}^{\text {disp }}(N)+\overline{\dot{\phi}}_{\text {dead }}^{\text {trans }}(N)=-\frac{k_{d}}{k} v_{p} \frac{\partial \bar{\phi}}{\partial N}+\frac{k_{c}}{k} \lambda \bar{\phi}(N) .
$$

The rhs of eq. (28) is calculated in Appendix F (see eq. (F5) using the expressions for $\phi$ derived in section 3.1 .

Thus collecting all terms, $\overline{\dot{\phi}}_{\text {dead }}=\bar{\phi}_{\text {dead }}^{\text {comb }}+\bar{\phi}_{\text {dead }}^{\text {disp }}+\bar{\phi}_{\text {dead }}^{\text {trans }}$, and noticing that the $k_{c} / k$ term of eq. (F5) cancels the last term of eq. (26), we have

$$
\overline{\dot{\phi}}_{\text {dead }}(N)=e^{-\lambda N / v_{p}}\left\{\bar{\phi}_{\text {dead }}^{\text {deal }}(N)+\varepsilon \dot{\phi}_{\text {dead }}^{\text {inc }}(N)\right\}, \quad \varepsilon \equiv \frac{4 \lambda}{(1-\beta) k \Psi_{0}}=\frac{4 \lambda \beta t_{0}}{(1-\beta)^{2}},
$$

where

$$
\begin{aligned}
\bar{\phi}_{\text {dead }}^{\text {inc }}(N) \equiv C \quad & {\left[\frac { k _ { c } } { k } \left\{\beta^{L}[(L+1) \beta-L]+\beta^{L}(L+1)(1-\beta) f\left(\frac{r N}{2}\right)\right.\right.} \\
& \left.\left.+L \beta^{L-1}(1-\beta) f\left(\frac{r N+n_{0}}{2}\right)\right\}+2 \frac{k_{d}}{k} \beta^{L} f(r N)\right] .
\end{aligned}
$$

The two contributions to the dead MWD, $\phi_{\text {dead }}=t \overline{\dot{\phi}}_{\text {dead }}$, are plotted in fig. [6] The incoherent part due to transfer events has no discontinuities, i. e. $\bar{\phi}_{\text {dead }}\left(L n_{0}^{-}\right)=\bar{\phi}_{\text {dead }}\left(\operatorname{lnc}\left(\operatorname{Ln}_{0}^{+}\right)\right.$, as may be seen using eq. (18). It is proportional to the small parameter $\varepsilon \approx \lambda t_{0}$.

Since the only discontinuous term in eq. (29) is $\bar{\phi}_{\text {dead }}^{\text {dideal }}$, using eq. (21) the discontinuity at $N=L n_{0}$ is

$$
\frac{\Delta \phi_{\text {dead }}\left(L n_{0}\right)}{\phi_{\text {dead }}(0)}=e^{-\lambda L t_{0}} \beta^{L} \frac{2+4 \rho}{1+4 \rho /(1-\beta)+\varepsilon(1+2 \rho)},
$$

where $\Delta \phi_{\text {dead }}\left(L n_{0}\right) \equiv \phi_{\text {dead }}\left(L n_{0}^{+}\right)-\phi_{\text {dead }}\left(L n_{0}^{-}\right)$. 


\section{Broadening and the Inflection Point Rule}

The MWD derived in the previous section, eq. (29), has ignored (i) Poisson broadening and (ii) broadening due to the limited accuracy of GPC measurements.

Consider first Poisson broadening. The true length distribution of a chain growing with mean velocity $v_{p}$ is not $\delta\left(N-v_{p} t\right)$ as has been assumed in sections 2 and 3, but rather the Poisson distribution (see fig. (7) which for large $N$ tends to a Gaussian:

$$
P_{t}(N)=\frac{\left(v_{p} t\right)^{N}}{N !} e^{-v_{p} t} \approx c e^{-\left(N-v_{p} t\right)^{2} /\left(2 v_{p} t\right)}, \quad(N \gg 1)
$$

Here $c=\left(2 \pi v_{p} t\right)^{-1 / 2}$ is a normalization constant.

Now since the termination rate constant $k$ is independent of chain length, clearly broadening leaves the total number of living chains $\Psi(t)$ unchanged. Hence the field $H(t)$ is unchanged, and thus a group of living chains injected at $t=0$ are still depleted in number by the factor $e^{-\int_{0}^{t} H}$ after time $t$, regardless of which chains are a little longer than $v_{p} t$ and which a little shorter.

Moreover we show in Appendix $\mathrm{G}$ that even in the presence of chain transfer, the Poisson broadened living MWD, $\phi_{\text {broad }}(N)$, can be expressed in terms of the unbroadened living $\mathrm{MWD}, \phi(N)$, calculated in the previous sections as follows:

$$
\phi_{\text {broad }}(N, t)=\int_{-\infty}^{\infty} d M \phi(M, t) \Delta(N ; M), \quad \Delta(N ; \bar{N}) \equiv \frac{1}{\sqrt{2 \pi \bar{N}}} e^{-(N-\bar{N})^{2} /(2 \bar{N})}
$$

Thus the $\delta$-pulses of $\phi$ in eq. (14) (or of $\phi_{c o h}$ in eq. (24) if chain transfer is present) are replaced by $\Delta$ 's, namely Gaussians of mean $\bar{N}$ and width $\sqrt{N}$. Note that with increasing $\bar{N}$, the relative width of the broadened $\Delta$-functions decreases as $1 / \sqrt{\bar{N}}$.

Then substituting eq. (33) into eq. (4) we find that the dead MWD, $\phi_{\text {dead }}^{\text {broad }}$, is also obtained by integrating the dead MWD in the absence of Poisson broadening, eq. (29), against a $\Delta$ function:

$$
\phi_{\text {dead }}^{\text {broad }}(N)=\int_{-\infty}^{\infty} d M \phi_{\text {dead }}(M) \Delta(N ; M)
$$

(We used the identity $\int_{-\infty}^{\infty} d M \Delta(M ; P) \Delta(N-M ; L)=\Delta(N ; L+P)$ for the termination by combination term of eq. (4)). Here $\phi_{\text {dead }}=t \overline{\dot{\phi}}_{\text {dead }}$. An example of the broadened MWD is shown in fig. [8 where $\phi_{\text {dead }}^{\text {broad }}$ is calculated by numerical integration of eq. (34). Eq. (34) has been called "a posteriori Poisson broadening".21] In the present work we proved its validity.

Now the accuracy with which the resulting dead MWD of eq. (34) is measured is limited by the resolution of the GPC equipment. Thus the MWD implied by the GPC output reading is

$$
\phi_{\text {dead }}^{\text {read }}(N)=\int_{-\infty}^{\infty} d M \phi_{\text {dead }}^{\text {broad }}(M) G(N ; M)
$$

where $G(N ; M)$ is the experimental reading (normalized to unity) given a perfectly monodisperse MWD input of chains of length $M$.

Consider values of $N$ near the discontinuity of the unbroadened dead MWD, $N=L n_{0}$, and let us examine the location of the inflection point. In the following we will assume that near the discontinuity to leading order $G$ can be approximated by a Gaussian distribution, $G(N ; n)=\left(2 \pi \sigma^{2}\right)^{-1 / 2} e^{-(N-n)^{2} /\left(2 \sigma^{2}\right)}$, where $\sigma$ is a measure of the experimental resolution near $L n_{0}$. Substituting $\phi_{\text {dead }}^{\text {broad }}$ from eq. (34) in eq. (35) one has

$$
\phi_{\text {dead }}^{\text {read }}(N)=\int_{-\infty}^{\infty} d M \phi_{\text {dead }}(M) \widetilde{\Delta}(N ; M), \quad \widetilde{\Delta}(N, M) \equiv \frac{e^{-(N-M)^{2} /\left[2\left(M+\sigma^{2}\right)\right]}}{\left[2 \pi\left(M+\sigma^{2}\right)\right]^{1 / 2}},
$$

after using $\int_{-\infty}^{\infty} d P \Delta(P ; M) G(N ; P)=\widetilde{\Delta}(N ; M)$ for Gaussian $G$.

In Appendix $\mathrm{H}$ the curvature of $\phi_{\text {dead }}^{\text {read }}$ is evaluated close to a discontinuity (eq. (H4)). Setting this to zero one finds two roots, one irrelevant at $\delta N \equiv N-L n_{0} \approx 2\left(L n_{0}+\sigma^{2}\right)$, and one corresponding to the point of inflection at

$$
\delta N \approx\left\{\frac{\phi_{+}^{\prime}-\phi_{-}^{\prime}}{\phi_{+}-\phi_{-}}\left(L n_{0}+\sigma^{2}\right)-\frac{1}{2}\right\}, \quad \phi_{+/-} \equiv \phi_{\text {dead }}^{\text {ideal }}\left(\operatorname{Ln}_{0}^{+/-}\right), \quad \phi_{+/-}^{\prime} \equiv \frac{d \phi_{\text {dead }}^{\text {ideal }}}{d N}\left(\operatorname{Ln}_{0}^{+/ /-}\right),
$$


Now in PLP experiments, parameters are normally chosen such that the dead MWD decreases by a factor of order unity between successive peaks. Hence $\phi_{+}^{\prime}$ is of order $\phi_{+} / n_{0}$ and similarly for $\phi_{-}$. Since $\phi_{+}^{\prime}<\phi_{-}^{\prime}<0$ and $\phi_{+}>\phi_{-}>0$, and taking $L=\mathcal{O}(1)$, one has from eq. (37),

$$
\delta N \approx-A\left(1+B \sigma^{2} / n_{0}\right)
$$

where $A, B>0$ are constants of order unity. This theoretical result is valid provided $n_{0}^{-1 / 2} \ll 1$ and $\beta$ is of order unity. Note that $\delta N$ is always negative.

Consider first very good experimental resolution, $\sigma \ll n_{0}^{1 / 2}$. In this case the observed broadening is predominantly Poissonian. According to eq. (38), the displacement of the inflection point of the measured MWD away from $L n_{0}$ is then of order one monomer. This is an unimportant correction which is of the same order as the accuracy of the continuum description of the MWD employed in our analysis. We conclude that for this case the inflection point is the appropriate point from which to extract $k_{p}$.

Now when $\sigma \gg n_{0}^{1 / 2}$, the broadening of the measured MWD is dominated by the finite experimental resolution. In this case the inflection point is of order $\sigma^{2} / n_{0} \gg 1$ monomers away from $L n_{0}$. Therefore for large $\sigma$, using the location of the inflection point to deduce $n_{0}$ leads to a systematic underestimation of $v_{p}$. We remark however that this is more accurate than using the local minimum or maximum of the MWD which will then be at a distance of order $\sigma$ away from $n_{0}$ (since we have assumed that $\sigma \ll n_{0}$, hence $\left.\sigma \gg \sigma^{2} / n_{0}\right)$.

As a specific example, consider the experimental MWD of fig. 4. In this figure, the distance between the local minimum and maximum of the MWD around the inflection point near molecular weight 50,000 $\left(n_{0} \approx 500\right)$ is of order 200 monomers. In this case Poisson broadening would only account for a width $\approx n_{0}^{1 / 2} \approx 22$ monomers. Hence broadening appears to be mainly due to limited experimental accuracy and $\sigma \approx 100$. The relative error using the inflection point for the calculation of $v_{p}$ would then lead to an error of order $\sigma^{2} / n_{0}^{2} \approx 4 \%$.

\section{Conclusions}

In this work, we have calculated analytically the number MWD of dead chains produced by pulsed laser polymerization at low conversions. The following effects have been included: living chain termination by both combination and disproportionation; chain transfer of living chain radicals to the environment; broadening effects. Ignoring broadening, we have found the MWD is given by

$$
\phi_{\text {dead }}(N)=e^{-\lambda N / v_{p}}\left\{\phi_{\text {dead }}^{\text {ideal }}(N)+\varepsilon \phi_{\text {dead }}^{\text {inc }}(N)\right\}, \quad \varepsilon=\frac{4 \lambda \beta t_{0}}{(1-\beta)^{2}},
$$

where

$$
\begin{aligned}
& \phi_{\text {dead }}^{\text {ideal }}(N)=\text { const. } \quad\left[\frac{k_{c}}{k}\left\{(L+1) \beta^{L} f^{2}\left(\frac{r N}{2}\right)+L \beta^{L-1} f^{2}\left(\frac{r N+n_{0}}{2}\right)\right\}+4 \frac{k_{d}}{k}\left\{\frac{\beta^{L}}{1-\beta} f^{2}(r N)\right\}\right], \\
& \phi_{\text {dead }}^{\text {inc }}(N) \equiv \text { const. } \quad\left[\frac { k _ { c } } { k } \left\{\beta^{L}[(L+1) \beta-L]+\beta^{L}(L+1)(1-\beta) f\left(\frac{r N}{2}\right)\right.\right. \\
& \left.\left.+L \beta^{L-1}(1-\beta) f\left(\frac{r N+n_{0}}{2}\right)\right\}+2 \frac{k_{d}}{k} \beta^{L} f(r N)\right], \\
& f(n) \equiv \quad \frac{\beta}{\beta+(1-\beta) n / n_{0}} .
\end{aligned}
$$

Here $N \equiv L n_{0}+r N$, and "const." is a normalization constant. The dead MWD is discontinuous at integer multiples of $n_{0}$ and is a function of the following independent parameters: $n_{0}, \lambda t_{0}, k_{d} / k, k_{c} / k$, and $\beta$. Here $\beta$ (see eq. (13)), the fraction of living chains surviving between 2 successive pulses, is a function of the dimensionless parameter $k \bar{R}_{i} t_{0}^{2}$, where $\bar{R}_{i} t_{0}$ is the concentration of new radicals produced per pulse. Eq. (39) applies in the limit in which chain transfer is weak, i. e. $\lambda t_{0} \ll 1$. Setting $k_{c}=0$, eq. (39) reduces to the dead MWD derived in refs., 2324 while setting $\lambda=0$ one recovers the dead MWD derived in refs. $\frac{621}{62}$

An important quantity in eqs. (39) and (40) is the relative magnitude of the dead MWD discontinuities, shown in eq. (31). Optimally large discontinuities typically correspond to values of $\beta$ intermediate between 


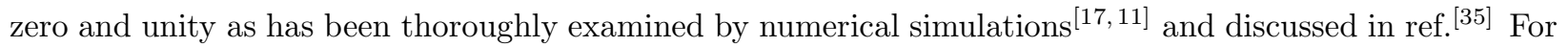
$\beta \ll 1$ these relative magnitudes are undesirably small. The limit $\beta \rightarrow 1$ (realised when $k \bar{R}_{i} t_{0}^{2}$ is small) may be undesirable also, but this depends on the mode of termination: (i) For the extreme of termination by disproportionation only ( $\rho \rightarrow \infty$ in eq. (31)) the discontinuities vanish in this limit while (ii) for termination by combination only $(\rho=0)$ the discontinuities attain their maximum value as $\beta \rightarrow 1$. Thus if combination is strongly dominant, by choosing small $R_{i}$ this may be a desirable limit (though noise effects are then particularly strong since the absolute amplitude of the dead MWD is very small). In a typical case though where termination occurs by both combination and disproportionation the optimal choice corresponds to intermediate $\beta$ values.

The effect of chain transfer is to reduce the amplitude of the entire MWD. The amplitude of the discontinuities is reduced by a factor $e^{-\lambda t_{0} N / n_{0}}$. (It follows that if $\lambda t_{0}>1$, most living chains suffer a chain transfer event before growing to a length of order $n_{0}$; consequently the MWD amplitude at $N=n_{0}$ is small. Under such conditions PLP is not a useful method for measuring $n_{0}$.) In addition to these effects, transfer introduces an extra "incoherent" contribution to the dead MWD (see fig. 6) which has no discontinuities and whose magnitude is a fraction $\lambda t_{0}$ of the dead MWD.

We have also treated 2 types of broadening: Poisson and distortion by MWD measuring equipment. Poisson broadening smoothes out the discontinuities of the dead MWD of eq. (39). The broadened dead MWD can be computed by performing numerically the integral of eq. (34). We found that for $\beta$ of order unity the position of the inflection point of the smooth MWD is not shifted significantly away from the initial discontinuity. ( For very small $\beta$ the inflection point rule leads to underestimation of $n_{0}$.) Thus, if Poisson broadening dominates, then its location can be used reliably to infer $k_{p}$.

In practice, the 2nd type of broadening, due to limited GPC resolution, may swamp the Poisson effect. We found that when the band width $\sigma$ of the GPC output reading, given a perfectly monodisperse sample of degree of polymerization $L n_{0}$, is much greater than $n_{0}^{1 / 2}$, experimental broadening then dominates near the discontinuity at $L n_{0}$. This leads to a displacement of the point of inflection by approximately $\sigma^{2} / n_{0}$ monomers below $L n_{0}$. We remark however that (for $\beta$ of order unity and $\sigma \ll n_{0}$ ) the point of inflection is closer to $n_{0}$ than the position of either the minimum or maximum peaks.

Acknowledgments. This work was supported by the National Science Foundation under grant no. DMR-9816374. We thank Erdem Karatekin for illuminating discussions. 


\section{A Solution to Living Chain Dynamics for Arbitrary $R_{i}(t)$}

In this appendix we solve eq. (6) for general radical production rate $R_{i}(t)$. Defining $\xi \equiv t-N / v_{p}$ and $f(\xi, t) \equiv \phi(N, t)$ we have from eq. (6)

$$
\left.\frac{\partial f}{\partial t}\right|_{\xi}=-H(t) f+\delta\left(v_{p} t-v_{p} \xi\right) R_{i}(t)
$$

with $\phi(N<0, t)=0$ implying $f(\xi>t)=0$. The solution of eq. A1 is

$$
f(\xi, t)=\frac{1}{v_{p}} \int_{-\infty}^{t} d t^{\prime} e^{-\int_{t^{\prime}}^{t} H} \delta\left(t^{\prime}-\xi\right) R_{i}\left(t^{\prime}\right)=\Theta(t-\xi) \frac{1}{v_{p}} e^{-\int_{\xi}^{t} H} R_{i}(\xi),
$$

where $\Theta$ is the step function, $\Theta(x>0)=1$ and $\Theta(x<0)=0$. Hence

$$
\phi(N, t)=\frac{\Theta(N)}{v_{p}} e^{-\int_{t-N / v_{p}}^{t} H\left(t^{\prime}\right) d t^{\prime}} R_{i}\left(t-N / v_{p}\right) .
$$

\section{B Properties of the Reaction Field $H(t)$}

Since $\dot{\Psi}=-H \Psi$ within one cycle (see eq. (9)), it follows that if both $t_{1}$ and $t_{2}$ belong to the same cycle then

$$
e^{-\int_{t_{1}}^{t_{2}} H\left(t^{\prime}\right) d t^{\prime}}=\Psi\left(t_{2}\right) / \Psi\left(t_{1}\right), \quad\left(\left[t_{1}\right]=\left[t_{2}\right]\right) .
$$

Since $\Psi(t)$ drops by a factor $\beta$ in one cycle of duration $t_{0}$ we also have

$$
e^{-\int_{t_{1}}^{t_{2}} H\left(t^{\prime}\right) d t^{\prime}}=e^{-\int_{\left[t_{1}\right] t_{0}}^{\left[t_{2}\right] t_{0}} H\left(t^{\prime}\right) d t^{\prime}} e^{-\int_{r t_{1}}^{r t_{2}} H\left(t^{\prime}\right) d t^{\prime}}=\frac{\Psi\left(t_{2}\right)}{\Psi\left(t_{1}\right)} \beta^{\left[t_{2}\right]-\left[t_{1}\right]}
$$

after using eq. (B1).

\section{General Expressions for Dead MWD's Generated by Combina- tion}

Consider two living MWDs $\phi_{1}, \phi_{2}$ which are periodic in time with period $t_{0}$ such that $\phi_{1}$ can be written as

$$
\phi_{1}(N, t)=\sum_{j=-\infty}^{\infty} A_{1}(N) f_{1}\left(N-v_{p} t-j n_{0}\right)
$$

where $f_{1}$ has period $n_{0}$. Suppose dead chains are generated only by bimolecular reactions involving one 1 and one 2 chain. If only combination reactions occur, the time averaged rate of dead chain generation then satisfies

$$
\begin{aligned}
\overline{\dot{\phi}}_{\text {dead }}(N) & =\frac{k}{t_{0}} \int_{0}^{t_{0}} d t \int_{-\infty}^{\infty} d M \phi_{1}(M, t) \phi_{2}(N-M, t) \\
& =\frac{k}{t_{0}} \sum_{j=-\infty}^{\infty} \int_{0}^{t_{0}} d t \int_{-\infty}^{\infty} d M A_{1}(M) f_{1}\left(M-v_{p} t-j n_{0}\right) \phi_{2}(N-M, t) \\
& =\frac{k}{t_{0}} \sum_{j=-\infty}^{\infty} \int_{j t_{0}}^{(j+1) t_{0}} d t^{\prime} \int_{-\infty}^{\infty} d M A_{1}(M) f_{1}\left(M-v_{p} t^{\prime}\right) \phi_{2}\left(N-M, t^{\prime}+j t_{0}\right)
\end{aligned}
$$


where we changed variables to $v_{p} t^{\prime}=v_{p} t+j n_{0}$. We remark that when there is only one chain type reacting with its own type to produce dead chains, then $\phi_{1}=\phi_{2}$ and the rhs of eq. (C2) should be divided by 2 . Since $\phi_{2}$ has period $t_{0}$ one has the following equivalent expression:

$$
\bar{\phi}_{\text {dead }}(N)=\frac{k}{t_{0}} \int_{-\infty}^{\infty} d t^{\prime} \int_{-\infty}^{\infty} d M A_{1}(M) f_{1}\left(M-v_{p} t^{\prime}\right) \phi_{2}\left(N-M, t^{\prime}\right) .
$$

With $A_{1}(N, t) \rightarrow \phi_{\text {env }}(N), f_{1} \rightarrow \delta, \phi_{2} \rightarrow \phi_{1}, k \rightarrow k_{c}$ and dividing by 2 this yields eq. (16) of the main text.

\section{Ideal Case: Combination Part of Dead Chain MWD, eq.}

Changing variables $n=2 v_{p} t+j n_{0}$ in eq. (16) and using eq. (14) to substitute for $\phi$ in eq. (16) one has

$$
\begin{aligned}
\bar{\phi}_{\text {dead }}^{\text {comb }}(N) & =\frac{k_{c}}{4 v_{p} t_{0}} \int_{-\infty}^{\infty} d n \sum_{j=-\infty}^{\infty} \phi_{\text {env }}\left(\frac{n+j n_{0}}{2}\right) \phi_{\text {env }}\left(\frac{n-j n_{0}}{2}\right) \delta(N-n) \\
& =\frac{k_{c}}{4 v_{p} t_{0}} \sum_{j=-\infty}^{\infty} \phi_{\text {env }}\left(\frac{N+j n_{0}}{2}\right) \phi_{\text {env }}\left(\frac{N-j n_{0}}{2}\right) .
\end{aligned}
$$

Writing $N=L n_{0}+r N$, with $L \equiv[N]$, we evaluate the sum of eq. (D1) by noting that since $\phi_{\text {env }}$ is zero for negative values of its arguments, only $-L \leq j \leq L$ contribute. Now using eq. (15) one has

$$
\phi_{\text {env }}\left(\frac{(L+j) n_{0}+r N}{2}\right) \phi_{\text {env }}\left(\frac{(L-j) n_{0}+r N}{2}\right)= \begin{cases}\beta^{L} \phi_{e n v}^{2}(r N / 2) & (L-j \text { even }) \\ \beta^{L-1} \phi_{e n v}^{2}\left(\left(r N+n_{0}\right) / 2\right) & (L-j \text { odd }) \\ & (-L \leq j \leq L)\end{cases}
$$

Noting that the number of even and odd $L-j$ values in the interval $-L \leq j \leq L$ is $L+1$ and $L$, respectively, and using eqs. (D2) and (D1), eq. (17) of the main text is derived after expressing $\phi_{e n v}$ in terms of $\Psi$ using eq. (15).

\section{E Derivation of Coherent-Incoherent Coupling Term $\bar{\phi}_{\text {dead,cross }}^{\text {comb }}$}

Using eqs. (24) and eq. (15), one has from eq. (25) after integrating over the $\delta$-function:

$$
\begin{aligned}
\bar{\phi}_{\text {dead }, \text { cross }}^{\text {comb }}(N) & =\frac{k_{c}}{v_{p} t_{0}} \int_{0}^{N} d M \phi_{\text {env }}(M)\left\{\lambda e^{-\lambda N / v_{p}} \Psi\left(M / v_{p}\right) \beta^{1+[N-2 M]+[M]}\right\} \\
& =\frac{\lambda k_{c} e^{-\lambda N / v_{p}}}{v_{p} t_{0}}(1-\beta) \int_{0}^{N} d M \Psi^{2}\left(M / v_{p}\right) \beta^{L+1+[r N-2 r M]} \\
N & \equiv L n_{0}+r N, \quad M \equiv P n_{0}+r M .
\end{aligned}
$$

Here we used the identity $-[N-2 M]=[2 M-N]+1$. The integrand of eq. [E1) is independent of $P$ and only depends on the relative magnitude of $r N$ with respect to $r M$. Since $O \leq P \leq L$ we may split $\int_{0}^{N}$ into $L$ integrals $\int_{0}^{r N / 2}+\int_{r N / 2}^{\left(r N+n_{0}\right) / 2}+\int_{\left(r N+n_{0}\right) / 2}^{n_{0}}$ corresponding to $P \leq L-1$, plus the $P=L$ term whose upper integration limit is $r N$ instead of $n_{0}$ :

$$
\begin{aligned}
\bar{\phi}_{\text {dead } \text { cross }}^{\text {comb }}(N)= & \frac{\lambda k_{c} e^{-\lambda N / v_{p}}}{v_{p} t_{0}}(1-\beta) \times \\
& \left\{(L+1) \beta^{L+1} \int_{0}^{r N / 2} d(r M) \Psi^{2}\left(r M / v_{p}\right)+(L+1) \beta^{L} \int_{r N / 2}^{\left(r N+n_{0}\right) / 2} d(r M) \Psi^{2}\left(r M / v_{p}\right)\right. \\
& \left.+L \beta^{L-1} \int_{\left(r N+n_{0}\right) / 2}^{n_{0}} d(r M) \Psi^{2}\left(r M / v_{p}\right)+\beta^{L} \int_{\left(r N+n_{0}\right) / 2}^{r N} d(r M) \Psi^{2}\left(r M / v_{p}\right)\right\}
\end{aligned}
$$




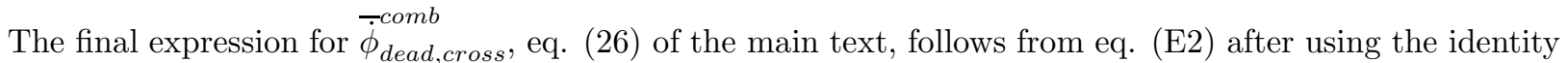
$-k \int_{t_{1}}^{t_{2}} d t \Psi^{2}=\Psi\left(t_{2}\right)-\Psi\left(t_{1}\right)$ for $0<t_{1}, t_{2}<t_{0}$.

\section{F Disproportionation and Chain Transfer Contribution to Dead MWD}

From eq. (24), the time average of $\phi_{c o h}$ is

$$
\bar{\phi}_{c o h}(N)=\frac{1}{v_{p} t_{0}} e^{-\lambda N / v_{p}} \phi_{e n v}(N) .
$$

Hence using the definition of $\phi_{\text {env }}$ (eq. (15)) and $\dot{\Psi}(t)=-k \Psi^{2}(t)$ for $0<t<t_{0}$,

$$
-v_{p} \frac{\partial \bar{\phi}_{c o h}}{\partial N}=\frac{1}{v_{p} t_{0}} e^{-\lambda N / v_{p}} \phi_{e n v}(N)\left[\lambda+k \Psi\left(r N / v_{p}\right)\right]
$$

The time average of $\phi_{i n c}$ is calculated after integrating the $3 \mathrm{rd}$ expression in eq. (24) from 0 to $t_{0}$. The value of the integrand depends on the relative magnitudes of $r N / v_{p}$ and $t$ :

$$
\bar{\phi}_{i n c}(N)=\frac{\lambda e^{-\lambda N / v_{p}}}{v_{p} t_{0}} \beta^{[N]+1}\left\{\int_{0}^{r N / v_{p}} d t \Psi(t)+\frac{1}{\beta} \int_{r N / v_{p}}^{t_{0}} d t \Psi(t)\right\} .
$$

Hence neglecting terms proportional to $\lambda^{2}$,

$$
-v_{p} \frac{\partial \bar{\phi}_{i n c}}{\partial N}=\frac{1}{v_{p} t_{0}} \lambda e^{-\lambda N / v_{p}} \phi_{e n v}(N)+\mathcal{O}\left(\lambda^{2}\right)
$$

Thus from eqs. (F1)- (F4) one has

$$
-\frac{k_{d}}{k} v_{p} \frac{\partial \bar{\phi}}{\partial N}+\frac{k_{c}}{k} \lambda \bar{\phi}(N)=C e^{-\lambda N / v_{p}}\left\{4 \frac{k_{d}}{k} \frac{\beta^{[N]}}{1-\beta} f^{2}(r N)+\varepsilon\left(2 \frac{k_{d}}{k}+\frac{k_{c}}{k}\right) \beta^{[N]} f(r N)\right\},
$$

after using eqs. (15) and (18) and the definition of $\varepsilon$ in eq. (29). Here we neglected $\mathcal{O}\left(\lambda^{2}\right)$ terms.

\section{G Poisson Broadening of Living MWD: Derivation of eq.}

In order to include broadening effects in the living dynamics, a "diffusion" term must be added in eqs. (6) and (22) as follows:

$$
\dot{\phi}_{\text {broad }}=-v_{p} \frac{\partial \phi_{\text {broad }}}{\partial N}+\frac{v_{p}}{2} \frac{\partial^{2} \phi_{\text {broad }}}{\partial N^{2}}-\widetilde{H}(t) \phi+\widetilde{R}_{i}(t) \delta(N),
$$

where $\widetilde{H}(t) \equiv H(t)+\lambda$ and $\widetilde{R}_{i} \equiv R_{i}(t)+\lambda \Psi(t)$. In the absence of the last two sink and source terms, eq. (G1) describes the evolution of the probability distribution function a unidirectional walk with step size unity and velocity $v_{p}$, in the continuum limit. The diffusion coefficient for such a walk ${ }^{[36]}$ is $v_{p} / 2$.

Now in the absence of the source and sink terms, the propagator of eq. (G1) for a pulse generated at $N=0$ at $t=0$, is simply $\Delta\left(N ; v_{p} t\right)$. (Here we neglected the condition $\phi_{\text {broad }}(N<0 ; t)=0$ which implies that the propagator be zero for negative $N$ values. However for $v_{p} t>1$, the amplitude of $\Delta\left(N ; v_{p} t\right)$ becomes exponentially small for negative $N$. This implies that the use of $\Delta$ is a very good approximation provided we examine the living MWD for $N \gg 1$.) Hence the solution of eq. (G1) is

$$
\phi_{\text {broad }}(N, t)=\int_{-\infty}^{t} d t^{\prime} e^{-\int_{t^{\prime}}^{t} \widetilde{H}} R_{i}\left(t^{\prime}\right) \Delta\left(N ; v_{p}\left(t-t^{\prime}\right)\right) .
$$

This solution is identical to the solution of the unbroadened living MWD in eq. (A2), after replacing $\delta\left(t^{\prime}-\xi\right)=v_{p} \delta\left(N-v_{p}\left(t-t^{\prime}\right)\right)$ in eq. (A2) by $\Delta\left(N ; v_{p}\left(t-t^{\prime}\right)\right)$. Hence eq. (33) follows immediately as one may check after replacing $\phi$ from eq. (A2) into eq. (33) and checking that eq. (G2) is recovered. 


\section{H Curvature of Broadened Dead MWD Near the Point of Inflec- tion}

Considering molecular weights near $L n_{0}$, expanding $\phi_{\text {dead }}(n)$ one has (see eq. (37) for notation)

$$
\phi_{\text {dead }}(n)=\left(\phi_{+}+\phi_{+}^{\prime} \delta n+\ldots\right) \Theta(\delta n)+\left(\phi_{-}+\phi_{-}^{\prime} \delta n+\ldots\right) \Theta(-\delta n), \quad \delta n \equiv n-L n_{0} \quad .
$$

Since the amplitude of the unbroadened dead MWD decreases smoothly by a factor of order unity between successive peaks, the magnitude of $\phi_{+}^{\prime}$ is of order $\phi_{+} / n_{0}$, and similarly for $\phi_{-}^{\prime}$. Now since the $\delta n$ which will survive in the integration of eq. (34) around $N \approx L n_{0}$ is of order $\Sigma \equiv\left(L n_{0}+\sigma^{2}\right)^{1 / 2}$, eq. (H1) is an

expansion up to order $\Sigma / n_{0}$. Approximating $\widetilde{\Delta}$ with a Gaussian of fixed width, and keeping terms up to order $1 / \Sigma\left(\right.$ note $\left.\Sigma / n_{0} \geq 1 / \Sigma\right)$ :

$$
\begin{aligned}
\widetilde{\Delta}(N ; n) & =\frac{1}{\left(2 \pi \Sigma^{2}\right)^{1 / 2}} e^{-(\delta N-\delta n)^{2} /\left(2 \Sigma^{2}\right)}\left\{1-\frac{\delta n}{2 \Sigma^{2}}+\frac{\delta n(\delta N-\delta n)^{2}}{2 \Sigma^{4}}+\ldots\right\} \\
\delta N & \equiv N-L n_{0}
\end{aligned}
$$

Substituting eqs. (H1) and (H2) in eq. (34) we obtain the following approximation to the MWD output reading near $L n_{0}$ :

$$
\begin{aligned}
\phi_{\text {dead }}^{\text {read }}(N) & =\frac{1}{\left(2 \pi \Sigma^{2}\right)^{1 / 2}} \int_{0}^{\infty} d(\delta n)\left\{\phi_{+}+\delta n\left(\phi_{+}^{\prime}-\frac{\phi_{+}}{2 \Sigma^{2}}+\frac{\phi_{+}(\delta N-\delta n)^{2}}{2 \Sigma^{4}}\right)\right\} e^{-(\delta N-\delta n)^{2} /\left(2 \Sigma^{2}\right)} \\
& +\frac{1}{\left(2 \pi \Sigma^{2}\right)^{1 / 2}} \int_{-\infty}^{0} d(\delta n)\{\ldots\}_{\phi_{+}, \phi_{+}^{\prime} \rightarrow \phi_{-}, \phi_{-}^{\prime}}
\end{aligned}
$$

A straightforward differentiation of eq. [H3) gives

$$
\frac{d^{2} \phi_{\text {dead }}^{\text {read }}}{d N^{2}}=\frac{1}{\left(2 \pi \Sigma^{2}\right)^{1 / 2}}\left\{\phi_{+}^{\prime}-\phi_{-}^{\prime}+\frac{\phi_{-}-\phi_{+}}{2 \Sigma^{2}}\left[1+2 \delta N-\frac{(\delta N)^{2}}{\Sigma^{2}}\right]\right\} e^{-(\delta N)^{2} /\left(2 \Sigma^{2}\right)} .
$$




\section{References}

[1] P. Flory, Principles of Polymer Chemistry, Cornell University Press, Ithaca, New York 1971.

[2] G. Odian, Principles of Polymerization, John Wiley and Sons, New York 1981.

[3] M. Buback, , R. G. Gilbert, R. A. Hutchinson, B. Klumperman, F.-D. Kuchta, B. Manders, K. F. O’Driscoll, G. T. Russell, J. Schweer, Macromol. Chem. Phys. 1995, 196, 3267-3280.

[4] S. Beueremann, M. Buback, Progress in Polymer Science 2002, 27, 191-254.

[5] M. L. Coote, M. D. Zammit, T. P. Davis, Trends in Polymer Science 1996, 4, 189-196.

[6] O. F. Olaj, I. Bitai, F. Hinkelmann, Makromol. Chem. 1987, 188, 1689-1702.

[7] R. A. Hutchinson, J. D. A. Paquet, J. H. McMinn, R. E. Fuller, Macromolecules 1995, 28, 4023-4028.

[8] R. A. Hutchinson, S. Beuermann, D. A. Paquet Jr., J. H. McMinn, C. Jackson, Macromolecules 1998, $31,1542-1547$.

[9] R. A. Hutchinson, S. Beuermann, D. A. Paquet Jr., J. H. McMinn, Macromolecules 1997, 30, 3490-3493.

[10] R. A. Hutchinson, M. T. Aronson, J. R. Richards, Macromolecules 1993, 26, 6410-6415.

[11] R. A. Hutchinson, J. R. Richards, M. T. Aronson, Macromolecules 1994, 27, 4530-4537.

[12] D. A. Shipp, T. A. Smith, D. H. Solomon, G. Moad, Macromol. Rapid Commun. 1995, 16, 837-844.

[13] R. A. Hutchinson, J. D. A. Paquet, J. H. McMinn, Macromolecules 1995, 28, 5655-5663.

[14] G. Zifferer, O. F. Olaj, Macromol. Theo. Simul. 1998, 7, 157-169.

[15] J. Sarnecki, J. Schweer, Macromolecules 1995, 28, 4080-4088.

[16] M. Busch, A. Wahl, Macromol. Theory Simul. 1998, 7, 217-224.

[17] M. Buback, M. Busch, R. A. Lammel, Macromol. Theory Simul. 1996, 5, 845-861.

[18] K. F. O'Driscoll, M. E. Kuindersma, Macromol. Theory Simul. 1994, 3, 469-478.

[19] D. Yan, M. Zhang, J. Schweer, Macromolecules 1996, 29, 3793-3799.

[20] A. P. Alexandrov, V. N. G. M. S. Kitai, I. M. Smirnova, V. V. Sokolov, Sov. J. Quantum Electron. (Engl. Transl.) 1977, 7(5), 547-550.

[21] A. Kornherr, G. Zifferer, O. F. Olaj, Macromol. Theory Simul. 1999, 8, 260-271.

[22] A. V. Evseev, A. N. Nikitin, Laser Chem. 1995, 16, 83-99.

[23] G. Zifferer, O. F. Olaj, Macromol. Theory Simul. 1996, 5, 901-921.

[24] G. Zifferer, O. F. Olaj, Macromol. Theory Simul. 1996, 5, 923-938.

[25] B. Friedman, B. O'Shaughnessy, Europhys. Lett. 1993, 23, 667-672.

[26] B. Friedman, B. O'Shaughnessy, Macromolecules 1993, 26, 5726-5739.

[27] B. Friedman, B. O'Shaughnessy, Int. J. Mod. Phys. B 1994, 8, 2555-2591.

[28] K. F. O’Driscoll, H. K. Mahabadi, J. Polym. Sci.: Polym. Chem. Ed. 1976, 14, 869-881.

[29] O. F. Olaj, A. Kornherr, G. Zifferer, Macromol. Rapid Commun. 1997, 18, 997-1007.

[30] O. F. Olaj, A. Kornherr, G. Zifferer, Macromol. Rapid Commun. 1998, 19, 89-96.

[31] O. F. Olaj, G. Zifferer, A. Korhnherr, Macromol. Theory Simul. 1998, 7, 321-326. 
[32] O. F. Olaj, A. Korhnherr, G. Zifferer, Macromol. Theory Simul. 1998, 7, 501-508.

[33] A. N. Nikitin, A. V. Evseev, Macromol. Theory Simul. 1997, 6, 1191-1210.

[34] A. N. Nikitin, A. V. Evseev, Macromol. Theory Simul. 1999, 8, 296-308.

[35] S. Beuermann, D. A. Paquet Jr., J. H. McMinn, R. A. Hutchinson, Macromolecules 1996, 29, 4206-4215.

[36] F. Reif, Fundamentals of Statistical and Thermal Physics, McGraw-Hill, New York 1965. 


\section{Figures}

FIG. 1. Schematic of monomer addition to the free radical end of a living chain.

FIG. 2. Termination reaction of 2 living chains of length $N$ and $M$. Combination results into one dead chain of length $N+M$ while disproportionation results into 2 dead chains of length $N$ and $M$.

FIG. 3. Schematic of a chain transfer reaction with monomer as the transfer agent.

FIG. 4. MWD of poly(methyl methacrylate) produced at $40^{\circ} \mathrm{C}$ by PLP with $t_{0}=.1$ sec as measured in ref. ${ }^{7}$ Horizontal axis is molecular weight. Arbitrary units on vertical axis.

FIG. 5. (a) Rate of radical production as a function of time. $R_{i}(t)$ is non-zero only during the short duration of the laser pulse the period of which is $t_{0}$. (b) Snapshot of the living chain MWD $\phi(N, t)$ during $0<t<t_{0}$ in the ideal case (absence of chain transfer and Poisson broadening). Here $\phi_{0} \equiv \phi(0)$. $\phi$ consists of a series of $\delta$-function pulses whose amplitude follows the envelope $\phi_{\text {env }}(N)$. (c) Number density of living chains as a function of time (see eq. (10)). (d) Dead MWD in the ideal case. $\phi_{\text {dead }}^{\text {ideal }}$ is discontinuous at integer multiples of $n_{0}$.

FIG. 6. Contributions to the dead MWD in the presence of chain transfer. The upper curve is the coherent contribution. The lower curve is the incoherent contribution, proportional to the small parameter $\varepsilon$. The slope of the incoherent MWD is discontinuous at integer multiples of $n_{0}$. Values of parameters used: $v_{p}=5000 \mathrm{~s}^{-1}, k_{c}=k_{d}=210^{7} \mathrm{M}^{-1} \mathrm{~s}^{-1}, \lambda=.5 \mathrm{~s}^{-1}, t_{0}=.1 \mathrm{~s}, \bar{R}_{i} t_{0}=510^{-7} \mathrm{M}$

FIG. 7. Snapshot of living MWD including the effects of Poisson broadening: the $\delta$-functions of the living MWD of fig. [5) are replaced by Gaussians.

FIG. 8. Illustration of dead MWD before and after broadening. The solid line is the unbroadened MWD. The dashed line is the Poisson broadened MWD. The dotted line is the reading on the GPC equipment, given the dashed line as the input MWD. We assumed uniform resolution $\sigma^{2}=5000$ (see main text). Values of parameters used: $v_{p}=5000 \mathrm{~s}^{-1}, k_{c}=k_{d}=510^{6} \mathrm{M}^{-1} \mathrm{~s}^{-1}, \lambda=.1 \mathrm{~s}^{-1}, t_{0}=.1 s, \bar{R}_{i} t_{0}=510^{-7} \mathrm{M}$. 


$$
\overbrace{\text { living }}^{\mathrm{N}}+\mathrm{O} \stackrel{\mathrm{k}_{\mathrm{p}}}{\mathrm{N}} \overbrace{\text { living }}^{\mathrm{N}+1} \bullet
$$




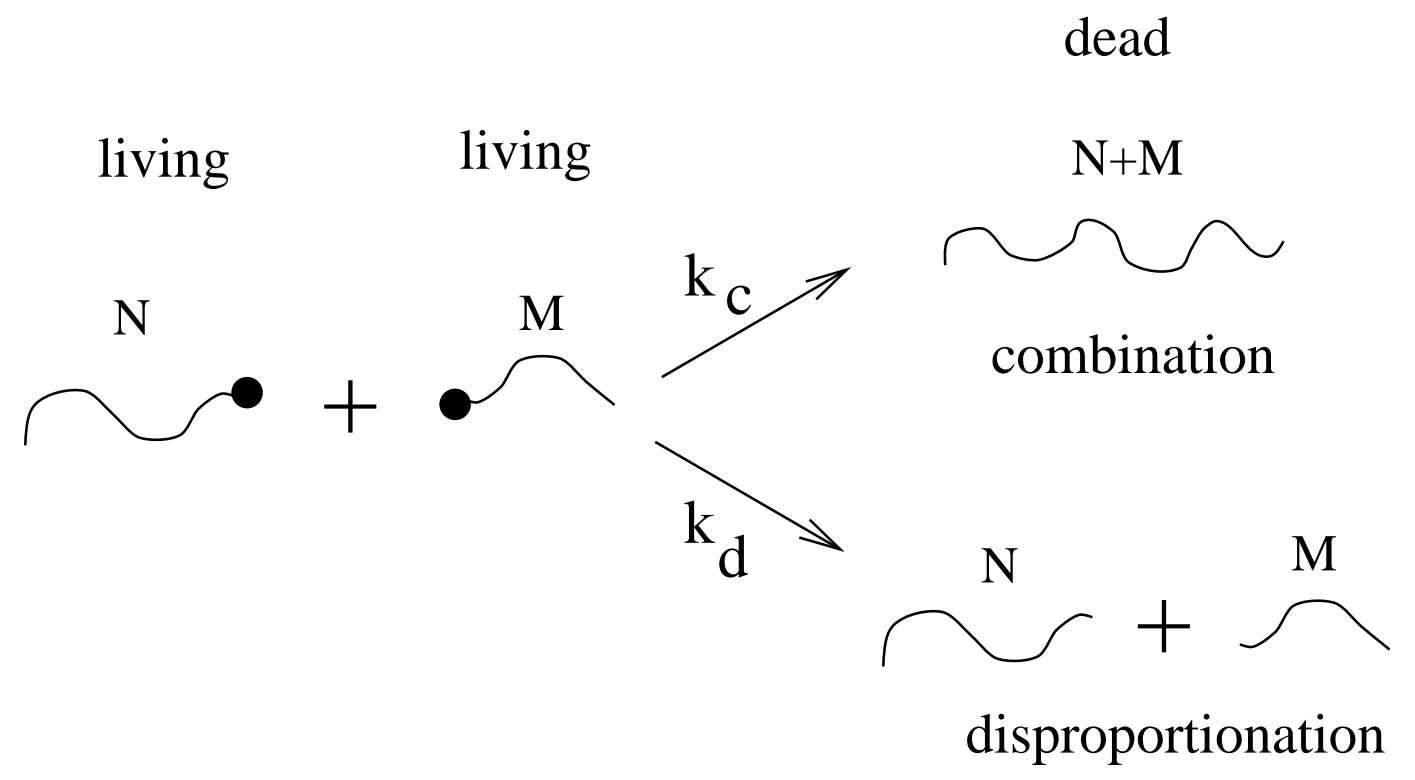

Fig.2 


$$
\begin{aligned}
& \bigcap^{N}+0 \stackrel{\lambda}{\longrightarrow} \overbrace{}^{N} \sim+\bullet \\
& \text { living monomer dead living } \\
& (\mathrm{N}=0)
\end{aligned}
$$




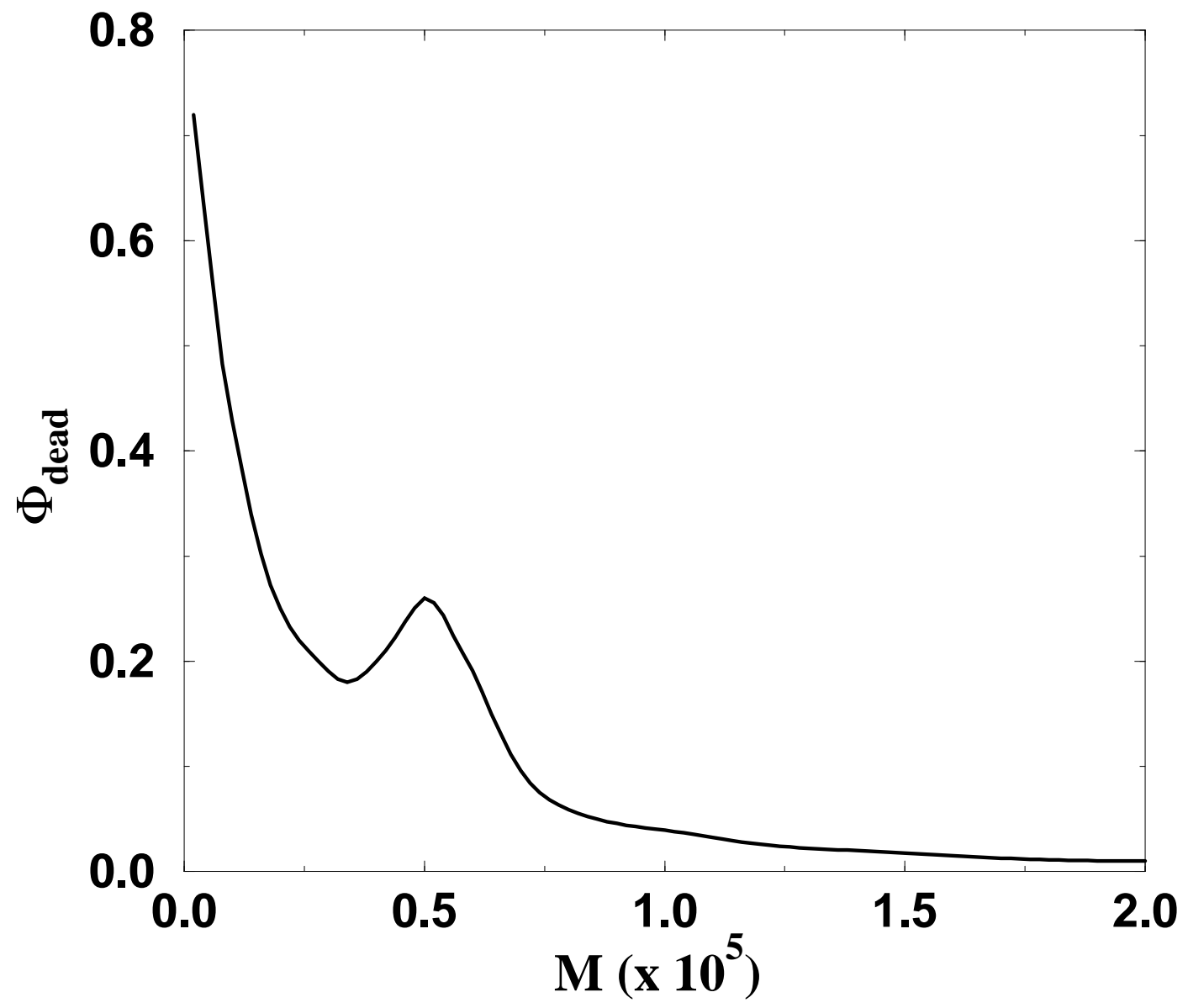

יד. 


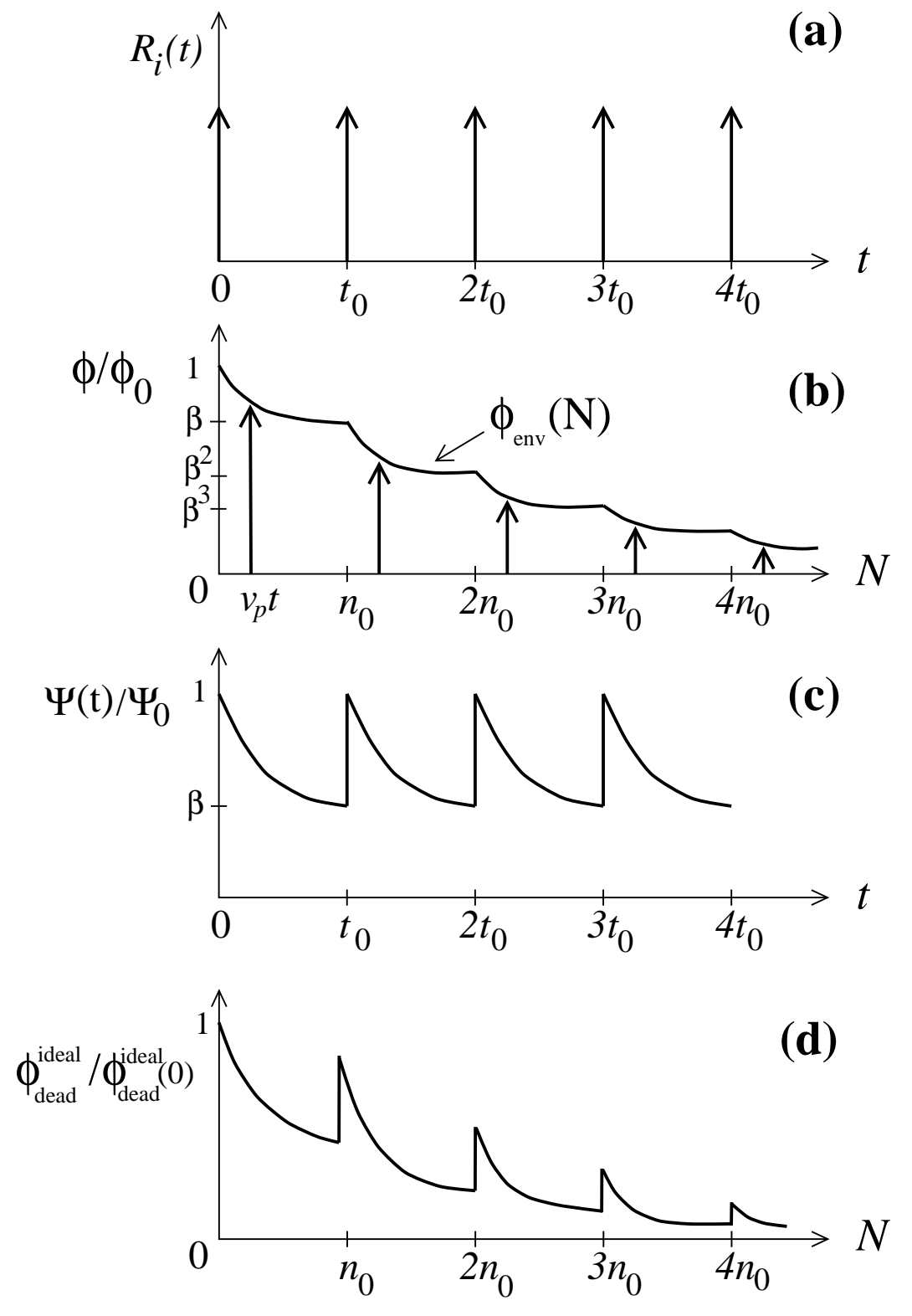

Fig.5 


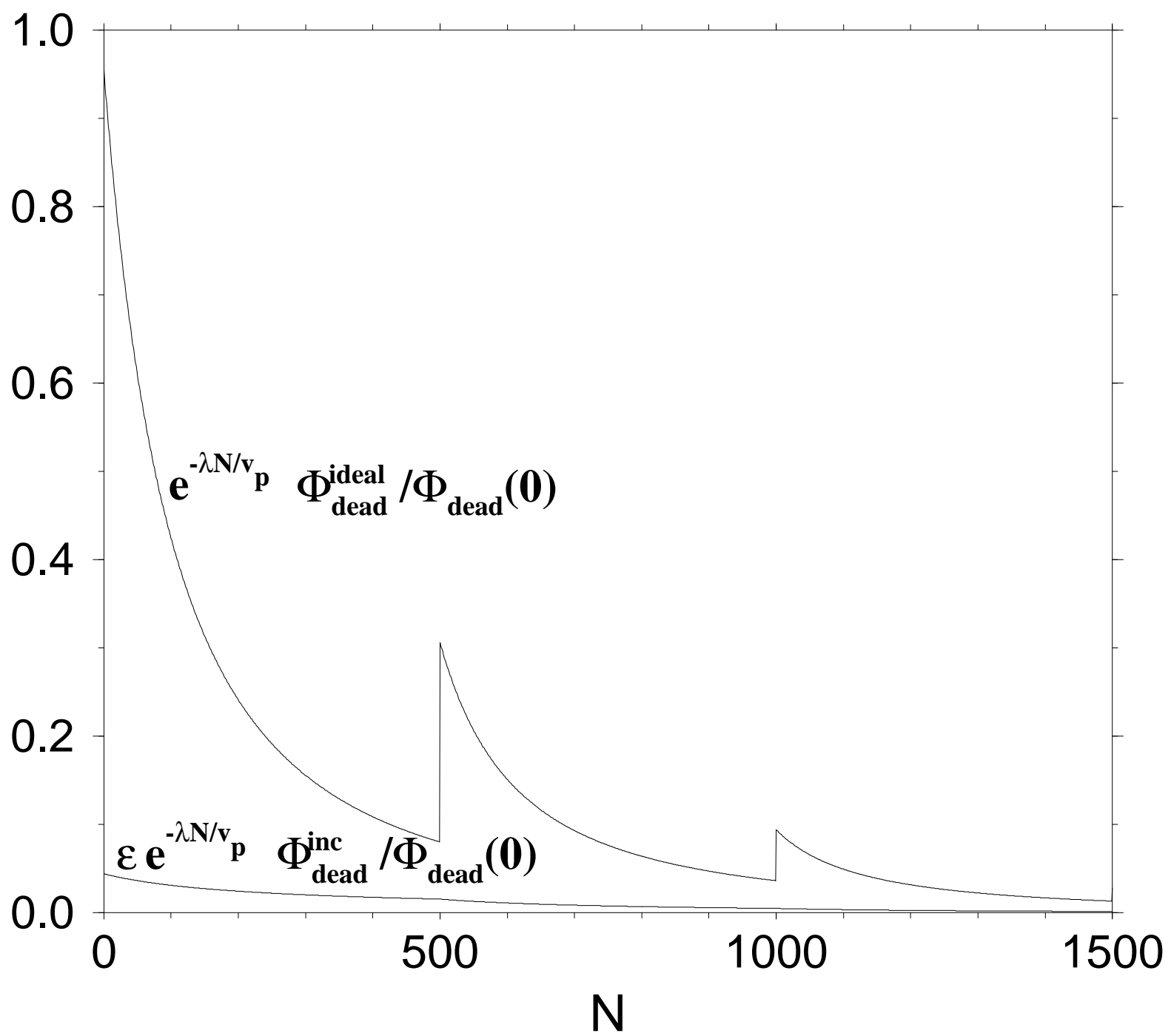

. 


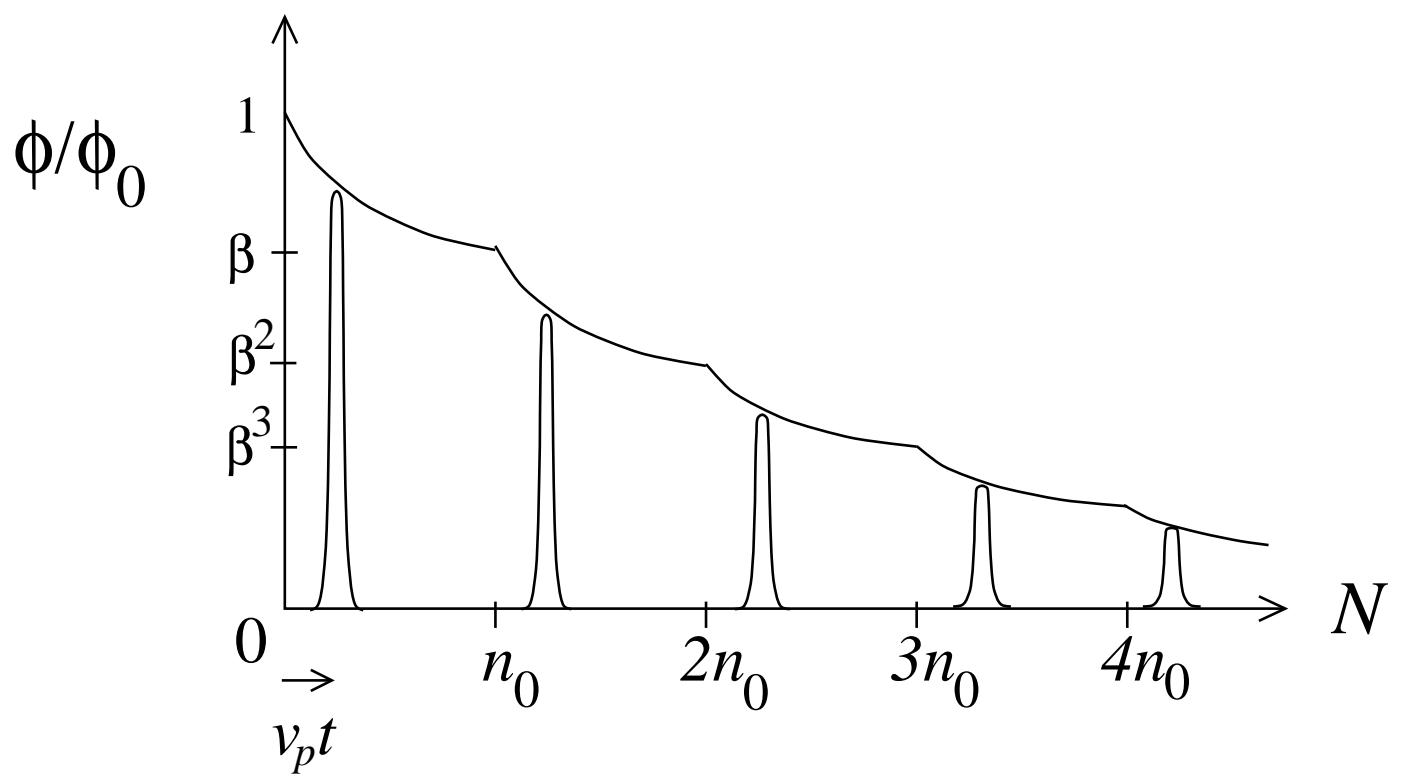

Fig.7 


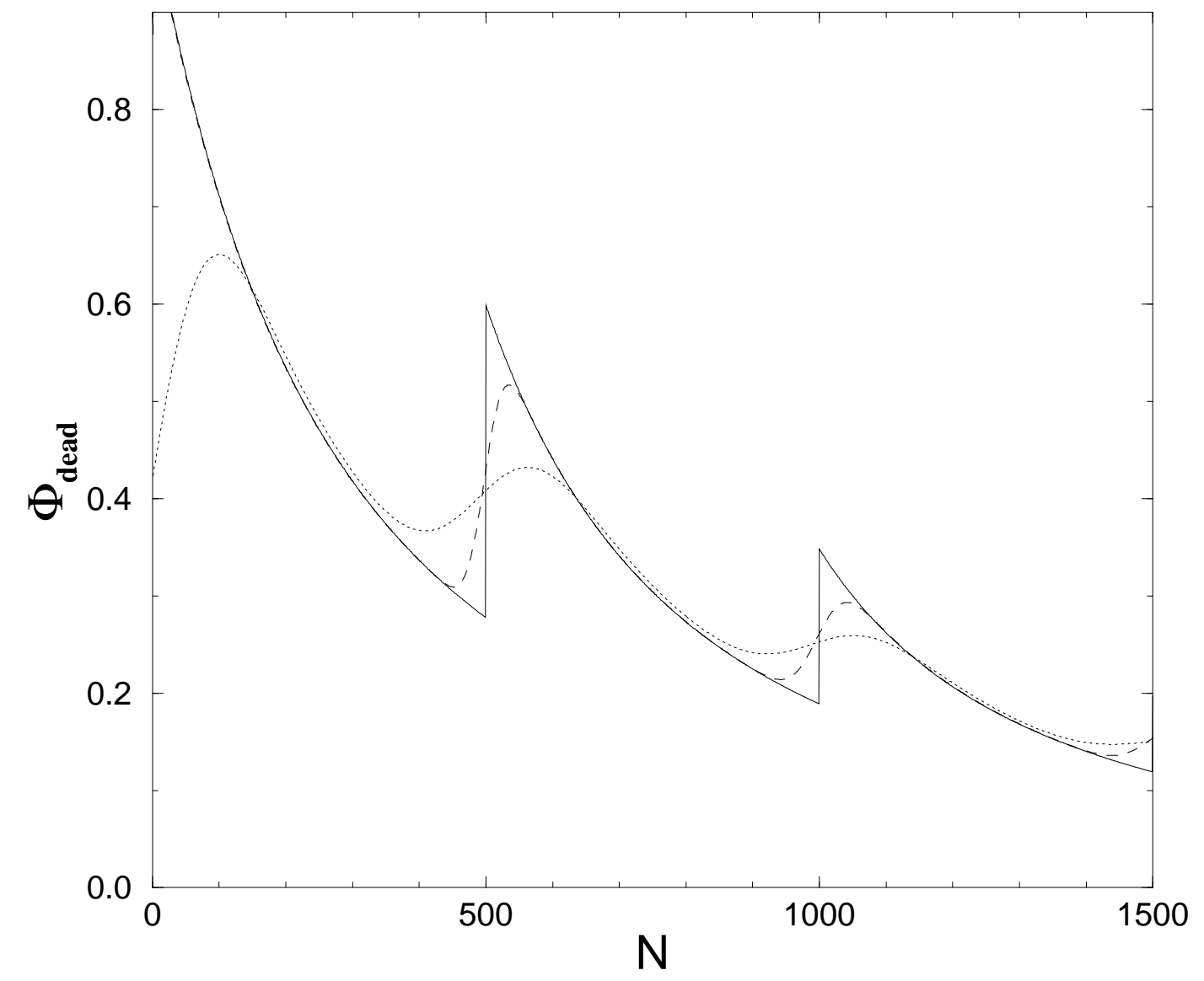

דיב 